\title{
Salutogenesis in the Era After Antonovsky
}

\author{
Shifra Sagy
}

Aaron Antonovsky was my mentor in the long journey of writing my doctoral dissertation, which was the first to be written in the framework of the salutogenic paradigm. He was not only my advisor in the academic research, but also had a tremendous impact on my life. For me, the salutogenic model is not only a theoretical paradigm whose genesis I witnessed and later on took an active part in its development. For me, this theory is the basis for a meaningful understanding of my lifestory, a story which has been embedded in the conflictual Jewish existence in Israel. Aaron and his salutogenic ideas have guided me in this difficult path too.

Aaron Antonovsky enriched us with a unique, challenging model, which had high levels of comprehensibility, manageability, and especially meaningfulness. When he passed away, 20 years ago, I wondered whether, and perhaps how, the model would be developed after him. Therefore, I am so deeply excited and enthusiastic to take part in this endeavor of the "Handbook of Salutogenesis" and especially pleased to edit this Part dealing with the era after him.

It was very tempting to continue Antonovsky's way by using his guidelines for salutogenic research (Antonovsky, 1996) and especially his concept of "sense of coherence" the SOC - as the primary answer for salutogenic questions. However, Aaron also taught me that "it is wise to see models, theories, constructs, hypotheses, and even ideas as heuristic devices, not only truths" (Antonovsky, 1996, p. 246). The chapters included in this Part represent good examples of this direction.

Chapter 6, written by Mittelmark, Bull, and Bouwman, focuses on some ideas which are examples of departures from traditional risk factor thinking. The models described

S. Sagy $(\bowtie)$

Head, Martin Springer Center for Conflict Studies, Ben-Gurion

University of the Negev, Beersheba, Israel

Department of Education, Ben-Gurion University of the Negev, Beersheba, Israel

e-mail: shifra@bgu.ac.il in this chapter were not aimed at continuing Antonovsky's model as a theory, but, it seems that the salutogenic paradigm has provided a useful foundation for these developments. This is quite clear in the Assets model in health promotion as well as in the Health Development Model. The other models described in this chapter (e.g., Fortigenesis, the Margins of Resources Model, the SelfTuning Model of Self-Care, Positive Deviance Approach) are other examples of the impact of salutogenic thinking, although in different directions. Perhaps it is the zeitgeist in health research that salutogenesis had been created which enabled these later developments.

The next two Chapters ( 7 and 8) are aimed at broadening our understanding of the salutogenic model by focusing on the important issue of resources. Idan, Eriksson, and Al-Yagon (Chap. 7) review and integrate conceptual and empirical research on the role of Generalized Resistance Resources (GRRs) within the salutogenic model. In particular, this chapter discusses findings regarding the conceptual and empirical progress in the study of GRRs at the individual, family, community, and ecological levels, which might enable us to understand individual differences in sense of coherence (SOC). Whereas this chapter focuses on the role of the GRRs in investigating SOC, the following chapter (Chap. 8) by Mittelmark, Bull, Daniel, and Urke focuses on the Specific Resistance Resources (SRRs) and discusses conceptual and concrete differences between generalized and specific resistance resources in the salutogenic model. This is important to health promotion research and practice, because the means by which these different types of resources are strengthened are dissimilar. The authors stress the importance of distinguishing between the two types of resistance resources, to ensure that health promotion pays balanced attention to both types. Generalized resistance resources arise from the cultural, social, and environmental conditions of living, and early childhood rearing and socialization experiences, in addition to idiosyncratic factors and chance, while the specific resistance resources are optimized 
by societal action in which health promotion has a contributing role. Taken together, this examination of both types of resources may provide a comprehensive understanding of the salutogenic model and the health promotion process.

The last two chapters in this section bring salutogenesis beyond health issues towards other areas of research. In Chapter 9, Joseph and Sagy attempt to integrate two paradigms - positive psychology and salutogenesis - and to suggest a joint conceptual framework which they term as "salutogenic positive psychology." Despite the differences between the two movements, and their different theoretical roots, we believe that the integrative approach has stronger explanatory power in promoting mental health and well-being.

In Chapter 10, Sagy and Mana wish to broaden the scope of the salutogenic paradigm into an interdisciplinary framework and to include other social concepts in its research. As one example of such interdisciplinary research, we review the new studies that investigate intergroup relations. By relating to such areas of research, we try to ask not only "who copes successfully and stays healthy?" but other salutogenic questions as well, such as, "who expresses more openness to the "other"?" I deeply believe that this meaningful question, stemming from our political and social reality, should also be discussed in the framework of salutogenesis.
Elsewhere in this handbook (Chap. 3, Antonovsky \& Sagy) we wrote that Aaron taught us that the most meaningful advancement in scientific work is to ask good questions. I trust that this Part of the Handbook relates well to this challenge, and end, how else, with Aaron's words, as he used to tell me at the end of our work meetings: "Let's start working; there is a lot of work to be done."

Open Access This chapter is distributed under the terms of the Creative Commons Attribution-Noncommercial 2.5 License (http:// creativecommons.org/licenses/by-nc/2.5/) which permits any noncommercial use, distribution, and reproduction in any medium, provided the original author(s) and source are credited.

The images or other third party material in this chapter are included in the work's Creative Commons license, unless indicated otherwise in the credit line; if such material is not included in the work's Creative Commons license and the respective action is not permitted by statutory regulation, users will need to obtain permission from the license holder to duplicate, adapt or reproduce the material.

\section{Reference}

Antonovsky, A. (1996). The salutogenic model as a theory to guide health promotion. Health Promotion International, 11(1), 11-18. 